\title{
THE AVERAGE FIRST RECURRENCE TIME
}

\author{
BY \\ BERNARD FRIEDMAN AND IVAN NIVEN
}

Introduction. The kinetic theory of gases contains a fundamental paradox in that the results obtained from the theory are irreversible despite the fact that gases are assumed to be composed of molecules which obey the reversible laws of mechanics. The paradox is further heightened by Poincaré's Cycle Theorem which states roughly that, no matter what is the initial configuration of the molecules, the gas must return to a state which is arbitrarily close to the initial state and that it must do so infinitely often. Physicists have tried to explain this paradox by stating that the time for one such Poincare cycle is so large that the recurrence of an initially improbable state is unlikely during the times normally available for observation. For a fuller discussion of these topics we refer to an article by Chandrasekhar [1].

In the present paper we consider the first recurrence time of a very simple dynamical system, namely, one which has $k+1$ degrees of freedom $x_{1}, x_{2}, \cdots, x_{k+1}$, each of which is simply-periodic in the time $\tau$. Put

$$
x_{j}(\tau)=A_{j} e^{2 \pi i v j \tau}, \quad j=1,2, \cdots, k+1 ;
$$

then the problem is to determine the smallest positive value of $\tau$ such that the point $\left(x_{1}(\tau), \cdots, x_{k+1}(\tau)\right)$ will be within a preassigned neighborhood of the initial point $\left(x_{1}(0), \cdots, x_{k+1}(0)\right)$. For simplicity, the neighborhood is assumed to be such that

$$
\left|\nu_{j} \tau-m_{j}\right| \leqq \epsilon, \quad j=1,2, \cdots, k+1,
$$

where $m_{1}, \cdots, m_{k+1}$ are integers. The smallest positive value of $\tau$ such that the inequalities (1) are satisfied for some set of integers will be called the first recurrence time of the system. Note that

$$
\tau=\tau\left(\nu_{1}, \cdots, \nu_{k+1} ; \epsilon\right) .
$$

We wish to find the average of the first recurrence time for all such systems, that is,

$$
\tau_{a v}=\int_{0}^{1} \cdots \int_{0}^{1} \tau\left(\nu_{1}, \cdots, \nu_{k+1} ; \epsilon\right) d \nu_{1} \cdots d \nu_{k+1}
$$

and in particular the asymptotic behavior of $\tau_{a v}$ as $\epsilon$ approaches zero.

Suppose that $\nu_{k+1} \neq 0$ is the largest of the $\nu_{j}$ and instead of the inequalities (1) consider the following:

$$
\nu_{k+1} \tau-m_{k+1}=0, \quad\left|\nu_{j} \tau-m_{j}\right|<\epsilon, \quad j=1,2, \cdots, \mathrm{k} .
$$

Presented to the Society October 27, 1956; received by the editors November 30, 1957. 
Put $\alpha_{j}=\nu_{j} / \nu_{k+1}, m_{k+1}=t$ then (3) becomes

$$
\left|t \alpha_{j}-m_{j}\right| \leqq \epsilon, \quad j=1,2, \cdots, k,
$$

where $t, m_{1}, \cdots, m_{k}$ are integers. We put

$$
t_{a v}=\int_{0}^{1} \cdots \int_{0}^{1} t\left(\alpha_{1}, \cdots, \alpha_{k} ; \epsilon\right) d \alpha_{1}, \cdots d \alpha_{k} .
$$

We shall show that there exist two positive constants $c_{1}$ and $c_{2}$ such that

$$
c_{1} \epsilon^{-k}<t_{a v}<c_{2} \epsilon^{-k} \text {. }
$$

For the case $k=1$, more can be obtained. We prove then that

$$
t_{a v}=\frac{6 \log 2}{\pi^{2}} \epsilon^{-1}+O(\log \epsilon),
$$

and

$$
\left\{t^{2}\right\}_{a v}=\frac{2 \log 2+1}{\pi^{2}} \epsilon^{-2}+O\left(\epsilon^{-1} \log \epsilon\right) .
$$

We wish to thank Professor Harold N. Shapiro for a suggestion which simplified the proofs of (6) and (7).

The one-dimensional case. For any $\alpha$ in the unit interval, define $t=t(\alpha, \epsilon)$ as the smallest positive integer such that $|t \alpha-m| \leqq \epsilon$ for some integer $m$, i.e., such that $t \alpha$ is within $\epsilon$ of an integer. Our problem is to evaluate

$$
\int_{0}^{1} t(\alpha, \epsilon) d \alpha
$$

Let the integer $n$ be chosen so that $1 /(n+1) \leqq \epsilon<1 / n$. Then it is clear that for any $\alpha$

$$
t\left(\alpha, \frac{1}{n}\right) \leqq t(\alpha, \epsilon) \leqq t\left(\alpha, \frac{1}{n+1}\right)
$$

and so

$$
\int_{0}^{1} t\left(\alpha, \frac{1}{n}\right) d \alpha \leqq \int_{0}^{1} t(\alpha, \epsilon) d \alpha \leqq \int_{0}^{1} t\left(\alpha, \frac{1}{n+1}\right) d \alpha
$$

Thus we examine

$$
\int_{0}^{1} t\left(\alpha, \frac{1}{n}\right) d \alpha
$$

Any real number $\alpha$ is said to have an admissible value $k$ (a positive integer) 
if $k \alpha$ is within $1 / n$ of an integer. Thus $t(\alpha, 1 / n)$ is the minimum of the admissible values for $\alpha$. Any rational number $a / b$ with $0 \leqq a / b \leqq 1$ defines an interval

$$
I\left(\frac{a}{b}\right)=\left(\frac{a}{b}-\frac{1}{n b}, \frac{a}{b}+\frac{1}{n b}\right),
$$

and all points in $I(a / b)$ have admissible value $b$. Furthermore $b$ is an admissible value for no real values of $\alpha$ other than those in the intervals $I(0 / b)$, $I(1 / b), \cdots, I(b / b)$.

Now if $t$ is the minimum integer such that $t \alpha$ is within $1 / n$ of an integer $m$, then $t \alpha-m=\delta / n$ with $|\delta| \leqq 1$. Thus $\alpha-m / t=\delta / t n$ and $m / t$ is in its lowest terms since otherwise $t /(t, m)$ would be an admissible value for $\alpha$ smaller than $t$ itself. Thus $\alpha \in I(m / t)$.

Let $F_{n}$ denote the Farey series of order $n$,

$$
F_{n}: \frac{0}{1}, \frac{1}{n}, \ldots, \frac{n-1}{n}, \frac{1}{1},
$$

that is, the series of rational numbers $a / b$ in ascending order satisfying $0 \leqq a \leqq b \leqq n$ with $(a, b)=1$. We use the following facts about Farey series [2, p. 23]: if $a_{1} / b_{1}$ and $a_{2} / b_{2}$ are two consecutive fractions in $F_{n-1}$ then $a_{1} b_{2}$ $-a_{2} b_{1}= \pm 1$ and $b_{1}+b_{2} \geqq n$.

Now if the Farey series $F_{n-1}$, of fractions $a / b$ say, is used to define a collection $C$ of intervals $I(a / b)$, we shall prove that $C$ covers the unit interval, but that no single interval $I(a / b)$ contains any member of $F_{n-1}$ other than $a / b$. From this it follows that to find $t(\alpha, 1 / n)$ we need merely locate $\alpha$ between two consecutive fractions $a_{1} / b_{1}$ and $a_{2} / b_{2}$ of $F_{n-1}$ and we have $t(\alpha, 1 / n)$ $=b_{1}$ if $\alpha$ is in $I\left(a_{1} / b_{1}\right)$ but not in $I\left(a_{2} / b_{2}\right) ; t(\alpha, 1 / n)=b_{2}$ if $\alpha$ is in $I\left(a_{2} / b_{2}\right)$ but not in $I\left(a_{1} / b_{1}\right) ; t(\alpha, 1 / n)=\min \left(b_{1}, b_{2}\right)$ if $\alpha$ is in both $I\left(a_{1} / b_{1}\right)$ and $I\left(a_{2} / b_{2}\right)$.

We prove the above assertions about the collection of intervals $C$. If $a_{1} / b_{1}$ and $a_{2} / b_{2}$ are adjacent fractions in $F_{n-1}$ then $a_{1} / b_{1}-a_{2} / b_{2}= \pm 1 / b_{1} b_{2}$ and since $b_{2}<n$ then $a_{2} / b_{2}$ is not in $I\left(a_{1} / b_{1}\right)$. To prove that $I\left(a_{1} / b_{1}\right)$ and $I\left(a_{2} / b_{2}\right)$ meet, (which will establish that $C$ covers the unit interval) we must prove that $1 / n b_{1}+1 / n b_{2} \geqq 1 / b_{1} b_{2}$. This follows from the fact that $b_{1}+b_{2} \geqq n$.

From this argument we see that the interval $\left(a_{1} / b_{1}, a_{2} / b_{2}\right)$, of length $1 / b_{1} b_{2}$, splits into two parts as regards the value of $t(\alpha, 1 / n): t(\alpha, 1 / n)$ $=\min \left(b_{1}, b_{2}\right)$ over a subinterval of length $1 / n \cdot \min \left(b_{1}, b_{2}\right), t(\alpha, 1 / n)$ $=\max \left(b_{1}, b_{2}\right)$ over one of length $1 / b_{1} b_{2}-1 / n \cdot \min \left(b_{1}, b_{2}\right)$. Thus

$$
\begin{aligned}
\int_{a_{1} / b_{1}}^{a_{2} / b_{2}} t\left(\alpha, \frac{1}{n}\right) d \alpha & =\frac{\min \left(b_{1}, b_{2}\right)}{n \cdot \min \left(b_{1}, b_{2}\right)}+\frac{\max \left(b_{1}, b_{2}\right)}{b_{1} b_{2}}-\frac{\max \left(b_{1}, b_{2}\right)}{n \cdot \min \left(b_{1}, b_{2}\right)} \\
& =\frac{1}{n}+\frac{n-\max \left(b_{1}, b_{2}\right)}{n \cdot \min \left(b_{1}, b_{2}\right)}
\end{aligned}
$$


since $b_{1} b_{2}=\min \left(b_{1}, b_{2}\right) \cdot \max \left(b_{1}, b_{2}\right)$. Let us denote $\min \left(b_{1}, b_{2}\right)$ by $b$ and $\max \left(b_{1}, b_{2}\right)$ by $B$, so that

$$
\int_{0}^{1} t\left(\alpha, \frac{1}{n}\right) d \alpha=\sum\left(\frac{1}{n}+\frac{n-B}{n b}\right)=\sum \frac{n+b-B}{n b}
$$

where the sum ranges over all pairs of consecutive fractions in $F_{n-1}$.

Lemma 1. Consider the set $S_{b}$ of all fractions in $F_{n-1}$ with fived denominator $b$, where $1 \leqq b \leqq n-2$. Let $T$ be the set of all fractions which are neighbors to members of $S_{b}$, and which have denominators exceeding $b$. If $B$ is any integer such that $(B, b)=1, b+B \geqq n, n-1 \geqq B \geqq b+1$, then the set $T$ has exactly two members with denominator $B$.

Proof. The equation $x b-y B=1$ has exactly one solution in integers $x$ and $y$ such that $1 \leqq x \leqq B-1,1 \leqq y \leqq b-1$. The fractions $x / B$ and $y / b$ are in $F_{n-1}$, and are neighbors, for if $y / b$ had the neighbor $h / k$ on the right, $y / b<h / k$ $<x / B$, then $h b-y k=1$ and $x b-y B=1$ imply that

$$
b(x k-h B)=k-B .
$$

But $x k-h B \geqq 1$, so that $k-B \geqq b$ and $k \geqq b+B \geqq n$, a contradiction.

Similarly the equation $X b-Y B=-1$ has exactly one solution $X, Y$ with $1 \leqq X \leqq B-1,1 \leqq Y \leqq b-1$ and likewise the fractions $X / B<Y / b$ are neighbors in $F_{n-1}$. Thus we have shown that the set $T$ contains at least two fractions with denominator $B$. On the other hand if $a / b$ and $A / B$ are neighbors in $F_{n-1}$, then $a B-A b= \pm 1$, so that the two cases we have examined are the only possible ones. Hence the lemma is proved.

By virtue of Lemma 1 we see that (8) can be written as

$$
\int_{0}^{1} t\left(\alpha, \frac{1}{n}\right) d \alpha=\frac{2}{n} \sum_{b=1}^{n-1} \sum_{B+b \geq n ; B=b+1 ;(B, b)=1}^{n-1} \frac{n+b-B}{b} .
$$

In what follows we shall use freely the following results: If $\mu(k)$ is the Möbius function, then

$$
\begin{aligned}
& \sum_{d \mid n} \mu(d)= \begin{cases}1, & \text { if } n=1 \\
0, & \text { otherwise }\end{cases} \\
& \sum_{d \mid n} \frac{\mu(d)}{d}=\frac{\phi(n)}{n}
\end{aligned}
$$

where $\phi(n)$ is the Euler function denoting the number of positive integers less than or equal to $n$ and prime to $n$.

We define

$$
\Phi(n)=\sum_{1}^{n} \phi(k)
$$


and

$$
T(n)=\sum_{b=1}^{n} \sum_{d \mid b} 1
$$

and we shall use the following properties of these functions:

Lemma 2.

$$
\begin{aligned}
\Phi(m) & =\frac{3 m^{2}}{\pi^{2}}+O(m \log m), \\
\sum_{1}^{m} \frac{\phi(k)}{k} & =\frac{6 m}{\pi^{2}}+O\left(\log ^{2} m\right), \\
\sum_{m+1}^{2 m} \frac{\phi(k)}{k^{2}} & =\frac{6 \log 2}{\pi^{2}}+O\left(\frac{\log m}{m}\right), \\
\sum_{1}^{m} k \phi(k) & =\frac{2 m^{3}}{\pi^{2}}+O\left(m^{2} \log m\right), \\
\sum_{m / 2 \leqq b \leqq m} b^{-1} \sum_{d \mid b} 1 & =O(\log m) .
\end{aligned}
$$

Proof. Statements (a) and (e) are in Hardy and Wright [2, p. 268 and Theorem 320]. Statement (c) is a special case of a result of D. H. Lehmer [3]. Statement (b) is derived from (a) as follows:

$$
\begin{aligned}
\frac{\phi(1)}{1}+\frac{\phi(2)}{2} & +\cdots+\frac{\phi(m)}{m} \\
& =\frac{\Phi(1)}{1}+\frac{\Phi(2)-\Phi(1)}{2}+\cdots+\frac{\Phi(m)-\Phi(m-1)}{m} \\
& =\sum_{j=1}^{m-1} \frac{\Phi(j)}{j(j+1)}+\frac{\Phi(m)}{m} \\
& =\frac{3}{\pi^{2}}\left\{\sum_{j=1}^{m-1} \frac{j^{2}}{j(j+1)}+\frac{m^{2}}{m}\right\}+O\left(\frac{m \log m}{m}\right)+\sum_{j=1}^{m-1} O\left(\frac{j \log j}{j(j+1)}\right) \\
& =\frac{3}{\pi^{2}}\{2 m+O(\log m)\}+O(\log m)+O\left(\log ^{2} m\right) \\
& =\frac{6 m}{\pi^{2}}+O\left(\log ^{2} m\right) .
\end{aligned}
$$


Statement (d) can also be derived from (a), thus,

$$
\begin{aligned}
\sum_{1}^{m} j \phi(j) & =m \Phi(m)-\sum_{1}^{m-1} \Phi(j) \\
& =\frac{3 m^{3}}{\pi^{2}}+O\left(m^{2} \log m\right)-\sum\left\{\frac{3 j^{2}}{\pi^{2}}+O(j \log j)\right\} \\
& =\frac{3 m^{3}}{\pi^{2}}-\frac{m^{3}}{\pi^{2}}+O\left(m^{2} \log m\right) .
\end{aligned}
$$

Finally, statement (f) follows from (e). We have

$$
\begin{aligned}
\sum_{[m / 2] \leqq b \leqq m} b^{-1} \sum_{d \mid b} 1 & =\sum \frac{T(b)-T(b-1)}{b} \\
& =\frac{T(m)}{m}-\frac{T([m / 2]-1)}{m / 2}+\sum_{[m / 2] \leqq b \leqq m-1} \frac{T(b)}{b(b+1)} \\
& =O\left(\sum_{[m / 2] \leqq b \leqq m} \frac{\log b}{b}\right) \\
& =O\left(\int_{m / 2}^{m} \frac{\log b}{b} d b\right) \\
& =O(\log m) .
\end{aligned}
$$

To finish the proof of (6), we return to formula (9) and observe that the sum can be written in the form

$$
\frac{2}{n} \sum_{b=1}^{n-1} \frac{1}{b} \sum_{B=b+1 ; B+b \geqq n}^{n-1}(n+b-B) \sum_{d \mid(B, b)} \mu(d) .
$$

We break this into two parts $T_{1}$ and $T_{2}$, where $T_{1}$ is the sum over values of $b$ satisfying $2 b<n$ and $T_{2}$ is the sum over $2 b \geqq n$. In $T_{1}$ we have

$$
2 b<n, n \geqq 2 b-1
$$

and so $B+b \geqq n$ implies

$$
B \geqq n-b \geqq b-1 ;
$$

therefore the sum ranges from $B=n-b$ to $B=n-1$ and we write

$$
T_{1}=\frac{2}{n} \sum_{b=1}^{[(n-1) / 2]} \frac{1}{b} \sum_{B=n-b}^{n-1}(n+b-B) \sum_{d \mid(B, b)} \mu(d) .
$$

In $T_{2}$ we have $2 b \geqq n$ so that $B \geqq b+1$ implies $B+b \geqq 2 b+1>n$; therefore the condition $B+b \geqq n$ is superfluous and we may write 


$$
T_{2}=\frac{2}{n} \sum_{b=[(n+1) / 2]}^{n-1} \frac{1}{b} \sum_{B=b+1}^{n-1}(n+b-B) \sum_{d \mid(B, b)} \mu(d) .
$$

Put $B=d s$ and interchange the order of the $B$ and $d$ summations. We get

$$
\begin{aligned}
T_{1} & =\frac{2}{n} \sum_{b=1}^{[(n-1) / 2]} \frac{1}{b} \sum_{d \mid b} d \mu(d) \sum_{s=[(n-b) / d]}^{[(n-1) / d]}\left(\frac{n+b}{d}-s\right) \\
& =\frac{2}{n} \sum_{b} \frac{1}{b} \sum_{d} d \mu(d)\left\{\frac{3 b^{2}}{2 d^{2}}+O\left(\frac{b}{d}\right)\right\} \\
& =\frac{2}{n} \sum_{b} \frac{3}{2} \phi(b)+\frac{2}{n} \sum_{b} \sum_{d} O(1) \\
& =\frac{3}{n} \sum_{b=1}^{[(n-1) / 2]} \phi(b)+O(\log n)
\end{aligned}
$$

by using (e) in Lemma 2 . Similarly we get

$$
\begin{aligned}
T_{2} & =\frac{2}{n} \sum_{b=[(n+1) / 2]}^{n-1} \frac{1}{b} \sum_{d \mid b} d \mu(d) \sum_{s=[(b+1) / d]}^{[(n-1) / d]}\left(\frac{n+b}{d}-s\right) \\
& =\frac{2}{n} \sum_{b} \frac{1}{b} \sum_{d} d \mu(d)\left\{\frac{n^{2}-b^{2}}{2 d^{2}}+O\left(\frac{n}{d}\right)\right\} \\
& =\frac{1}{n} \sum_{b} \frac{\left(n^{2}-b^{2}\right) \phi(b)}{b^{2}}+O(\log n)
\end{aligned}
$$

by using (f) in Lemma 2 .

We combine these results for $T_{1}$ and $T_{2}$ and use Lemma 2 to write

$$
\begin{aligned}
T_{1}+T_{2} & =\frac{3}{n} \sum_{b=1}^{[(n-1) / 2]} \phi(b)+n \sum_{[(n+1) / 2]}^{n-1} \frac{\phi(b)}{b^{2}}-\frac{1}{n} \sum_{[(n+1) / 2]}^{n-1} \phi(b)+O(\log n) \\
& =n \sum_{[(n+1) / 2]}^{n-1} \frac{\phi(b)}{b^{2}}+\frac{4}{n} \sum_{1}^{[(n-1) / 2]} \phi(b)-\frac{1}{n} \sum_{1}^{n-1} \phi(b)+O(\log n) \\
& =\frac{6 n \log 2}{\pi^{2}}+\frac{4 \Phi\left(\left[\frac{n-1}{2}\right]\right)-\Phi(n-1)}{n}+O(\log n) \\
& =\frac{6 n \log 2}{\pi^{2}}+O(\log n) .
\end{aligned}
$$

Having completed the proof of formula (6), we evaluate the second moment in the same way. The analysis preceding formula (8) is adapted to give 


$$
\begin{aligned}
\left\{t^{2}\right\}_{a v}= & \sum\left\{b^{2}\left(\frac{1}{n b}\right)+B^{2}\left(\frac{1}{b B}-\frac{1}{n b}\right)\right\} \\
= & \frac{2}{n} \sum_{b=1}^{[(n-1) / 2]} \frac{1}{b} \sum_{B=n-b}^{n-1}\left(b^{2}+B n-B^{2}\right) \sum_{d \mid(B, b)} \mu(d) \\
& +\frac{2}{n} \sum_{[(n+1) / 2]}^{n-1} \frac{1}{b} \sum_{B=b+1}^{n-1}\left(b^{2}+B n-B^{2}\right) \sum_{d \mid(B, b)} \mu(d) \\
= & S_{1}+S_{2} \text {, say. }
\end{aligned}
$$

When we interchange the order of summation with respect to $B$ and $d$ and put $B=s d$, sums of the form

$$
\sum_{s=\alpha}^{\beta}\left(b^{2}+n s d-s^{2} d^{2}\right)
$$

must be evaluated. This sum is equal to

$$
\begin{aligned}
b^{2}(\beta-\alpha)+n d \frac{\beta^{2}-\alpha^{2}}{2}-d^{2} \frac{\beta^{3}-\alpha^{3}}{3}+O\left(\beta^{2} d^{2}+\beta n d\right) \\
\quad=(\beta-\alpha)\left\{b^{2}+\frac{n d(\beta+\alpha)}{2}-\frac{d^{2}\left(\beta^{2}+\alpha \beta+\alpha^{2}\right)}{3}\right\}+O\left(n^{2}\right)
\end{aligned}
$$

since, as we shall see, $\beta d \leqq n$ in the application of this to both $S_{1}$ and $S_{2}$.

In $S_{1}$ the sum to be evaluated has $\alpha=[(n-b) / d], \beta=[(n-1) / d]$ and hence

$$
\begin{aligned}
S_{1} & =\frac{2}{n} \sum_{b=1}^{[(n-1) / 2]} \frac{1}{b} \sum_{d \mid b} \mu(d) \frac{b}{d}\left\{b^{2}+\frac{n}{2}(2 n-b)-\frac{3 n^{2}-3 n b+b^{2}}{3}+O\left(\frac{n^{2}}{b}\right)\right\} \\
& =\frac{2}{n} \sum_{b} \frac{\phi(b)}{b}\left\{\frac{2}{3} b^{2}+\frac{n b}{2}+O\left(\frac{n^{2}}{b}\right)\right\} \\
& =\frac{2}{n} \sum_{b} \frac{2}{3} b \phi(b)+\Phi\left(\left[\frac{n-1}{2}\right]\right)+\frac{2}{n} O\left(n^{2} \log n\right) .
\end{aligned}
$$

In $S_{2}$ the sum to be evaluated has $\alpha=[(b+1) / d], \beta=[(n-1) / d]$; hence

$$
\begin{aligned}
S_{2} & =\frac{2}{n} \sum_{b=[(n+1) / 2]}^{n-1} \frac{1}{b} \sum_{d \mid b} \mu(d) \frac{n-b}{d}\left\{b^{2}+\frac{n}{2}(n+b)-\frac{n^{2}+n b+b^{2}}{3}+O\left(\frac{n^{2}}{b}\right)\right\} \\
& =\frac{2}{n} \sum_{b} \frac{\phi(b)}{b^{2}}(n-b)\left\{\frac{2 b^{2}}{3}+\frac{b n}{6}+\frac{n^{2}}{6}+O\left(\frac{n^{2}}{b}\right)\right\} \\
& =\frac{2}{n} \sum_{b} \frac{\phi(b)}{b^{2}}\left\{\frac{n^{3}}{6}+\frac{n b^{2}}{2}-\frac{2 b^{3}}{3}+O\left(\frac{n^{3}}{b}\right)\right\} \\
& =\frac{2}{n} \sum_{b}\left\{\frac{n^{3} \phi(b)}{6 b^{2}}-\frac{2 b \phi(b)}{3}\right\}+\Phi(n-1)-\Phi\left(\left[\frac{n-1}{2}\right]\right)+O(n) .
\end{aligned}
$$


Combining $S_{1}$ and $S_{2}$ we get, by use of Lemma 2,

$$
\begin{aligned}
S_{1}+S_{2}= & \frac{n^{2}}{3} \sum_{b=[(n+1) / 2]}^{n-1} \frac{\phi(b)}{b^{2}}+\Phi(n-1)-\frac{4}{3 n} \sum_{b=1}^{n-1} b \phi(b) \\
& +\frac{8}{3 n} \sum_{b=1}^{[(n-1) / 2]} b \phi(b)+O(n \log n) \\
= & n^{2}\left\{\frac{2 \log 2}{\pi^{2}}+\frac{3}{\pi^{2}}-\frac{8}{3 \pi^{2}}+\frac{2}{3 \pi^{2}}\right\}+O(n \log n) \\
= & \frac{n^{2}}{\pi^{2}}(2 \log 2+1)+O(n \log n),
\end{aligned}
$$

and this completes the proof of (7).

For the higher dimensional cases we need the following inequality.

Lemma 3. Suppose the real numbers $A_{1}, A_{2}, \cdots, A_{m}$ and $B_{1}, B_{2}, \cdots, B_{r}$ with $m \geqq r$ satisfy the inequalities

$$
A_{1}+A_{2}+\cdots+A_{m} \geqq B_{1}+B_{2}+\cdots+B_{r}
$$

and $B_{i} \geqq A_{i}$ for $i=1,2, \cdots, r$. Then $A_{1}+2 A_{2}+3 A_{3}+\cdots+m A_{m} \geqq B_{1}+2 B_{2}$ $+3 B_{3}+\cdots+r B_{r}$.

Proof. For $m=1$ this result is trivial, and we proceed by induction. From the hypotheses we have $A_{2}+A_{3}+\cdots+A_{m} \geqq B_{2}+B_{3}+\cdots+B_{r}$ and we apply the result (in the case $m-1$ ) to the real numbers $A_{2}, \cdots, A_{m}$ and $B_{2}, \cdots, B_{r}$. Thus we have

$A_{2}+2 A_{3}+3 A_{4}+\cdots+(m-1) A_{m} \geqq B_{2}+2 B_{3}+3 B_{4}+\cdots+(r-1) B_{r}$, and by adding (10) we get the desired result.

To illustrate the higher dimensional cases, we take the two dimensional situation. The integer $b$ is an admissible value for all pairs of real numbers lying in the region defined by the Cartesian product of any interval

$$
I(0 / b), I(1 / b), \cdots, I(b / b)
$$

with any other. Any pair of real numbers outside this region does not have $b$ as an admissible value. These intervals have length $2 / n$ (disregarding what falls outside the unit interval of course) and the region defined by the Cartesian product has area $4 / n^{2}$. Thus if $A_{b}$ is the total area of the regions within the unit square for which any point $\left(\alpha_{1}, \alpha_{2}\right)$ has actual value $b$, i.e. for which

$$
t\left(\alpha_{1}, \alpha_{2}, 1 / n\right)=b
$$

then $A_{b} \leqq 4 / n^{2}$. Thus we have

$$
A_{1}+A_{2}+A_{3}+\cdots=1
$$


and we can apply Lemma 3 with each $B_{i}$ replaced by $4 / n^{2}$, taking $r=\left[n^{2} / 4\right]$. We conclude that

$$
\begin{aligned}
A_{1}+2 A_{2}+3 A_{3}+\cdots & \geqq \frac{4}{n^{2}}+2 \cdot \frac{4}{n^{2}}+\cdots+\left[\frac{n^{2}}{4}\right] \frac{4}{n^{2}} \\
& =\frac{4}{n^{2}}\left\{1+2+3+\cdots+\left[\frac{n^{2}}{4}\right]\right\} \\
& \geqq n^{2} / 9 \text { for } n \text { sufficiently large. }
\end{aligned}
$$

But by definition we have

$$
\int_{0}^{1} \int_{0}^{1} t\left(\alpha_{1}, \alpha_{2}, 1 / n\right) d \alpha_{1} d \alpha_{2}=A_{1}+2 A_{2}+3 A_{3}+\cdots \geqq n^{2} / 9 .
$$

In $k$ dimensions, this argument proves the existence of a positive constant $c_{1}$ such that

$$
\int_{0}^{1} \int_{0}^{1} \cdots \int_{0}^{1} t\left(\alpha_{1}, \alpha_{2}, \cdots, \alpha_{k}, 1 / n\right) d \alpha_{1} d \alpha_{2} \cdots d \alpha_{k} \geqq c_{1} n^{k} .
$$

This integral is less than $n^{k}$, because by the pigeon hole procedure we can find a positive integer $m \leqq n^{k}$ for any $\alpha_{1}, \cdots, \alpha_{k}$ such that $m \alpha_{1}, \cdots, m \alpha_{k}$ are all within $1 / n$ of an integer. Therefore, $t \leqq n^{k}$ and then

$$
\int_{0}^{1} \int_{0}^{1} \cdots \int_{\theta}^{1} t d \alpha_{1} d \alpha_{2} \cdots d \alpha_{k} \leqq n^{k}
$$

This completes the proof of (5).

\section{BiBLIOGRAPHY}

1. S. Chandrasekhar, Stochastic problems in physics and astronomy, Rev. Mod. Phys. vol. 15 (1943).

2. Hardy and Wright, Theory of numbers, 3d ed., Oxford, 1954.

3. D. H. Lehmer, A conjecture of Krishnaswami, Bull. Amer. Math. Soc. vol. 54 (1948) p. 1187.

University of California,

Berkeley, Calif.

UNIVERSITY OF OREGON,

EugENE, ORE. 